\title{
Elementos del pensamiento sistémico en la elaboración de explicaciones sobre el fenómeno de la caída de los cuerpos:
}

\author{
Clara Inés Chaparro Susa \\ Rosa Inés Pedreros Martínez \\ Néstor Fernando Méndez Hincapié \\ Hugo Orlando Sastoque Quevedo \\ César Augusto Prías Bastidas ${ }^{* *}$
}

Artículo recibido:31-03-2006 y Aprobado: 28-09-2006

System thinking elements in the construction of explanations about the phenomenon of falling bodies

\begin{abstract}
Resumen: Se busca consolidar una opción pedagógica y didáctica para la enseñanza de la física mediante la elaboración de explicación de los fenómenos naturales y el desarrollo del pensamiento sistémico de los estudiantes. En este trabajo se aportan elementos en la explicación de la caída de los cuerpos desde la perspectiva de los sistemas dinámicos en dos niveles de educación: básica y universitaria.
\end{abstract}

Palabras clave: sistemas dinámicos, pensamiento sistémico, enseñanza de la física, caída de los cuerpos.
Summary: In this paper we aim at showing how by means of constructing explanations to natural phenomena, and developing a systems thinking in students, a pedagogic and didactic alternative for teaching physics is consolidated. We also introduce some elements to explain the falling body from the point of view of Dynamic Systems in two educational levels: Elementary and University.

Key words: Dynamic Systems, Systems Thinking, Teaching Physics, Falling Body.

\footnotetext{
En este artículo se recogen reflexiones e indagaciones del proyecto “Los sistemas dinámicos y el pensamiento sistémico en el aula". Centro de Investigaciones de la Universidad Pedagógica Nacional, CIUP, código DFI 036- 04.

* Grupo Física y Cultura. Subgrupo Sistemas Dinámicos.

Profesores del Departamento de Física de la Universidad Pedagógica Nacional

chaparro@uni.pedagogica.edu.co

rpedreros@uni.pedagogica.edu.co

nmendez@uni.pedagogica.edu.co

hugosastoque@uni.pedagogica.edu.co

cprias@uni.pedagogica.edu.co
}

96 TEA N. ${ }^{0} 20$ • Segundo semestre de 2006 • pp. 96-109 


\section{Presentación}

El presente trabajo forma parte de las investigaciones realizadas por el equipo de Sistemas Dinámicos (SD) y responde a los proyectos llevados a cabo por la línea de investigación "Enseñanza de las ciencias desde una perspectiva cultural”, del Departamento de Física de la Universidad Pedagógica Nacional.

En el contexto de esta investigación, hacemos especial hincapié al desarrollo del pensamiento sistémico a través del trabajo con modelos de SD, el cual potencia en los estudiantes el desarrollo de un pensamiento sistemático o "interconectado", lo que les permite actuar y comprender el mundo contemporáneo de las ciencias naturales, en un momento en el que todo lo que se hace en un lugar tiene una influencia global. De ahí la importancia de que la escuela participe en la formación de un pensamiento complejo, acorde con las exigencias de un mundo también complejo.

En la construcción de estrategias pedagógicas que propicien el desarrollo de un pensamiento sistémico en nuestros estudiantes y maestros, el equipo de trabajo abordó el fenómeno de la caída de los cuerpos con estudiantes de enseñanza básica y universitaria, arrojando elementos para comprender las condiciones pedagógicas que hagan posible la elaboración de explicaciones de los propios estudiantes en las que se involucren las cuatro características del pensamiento sistémico: pensamiento en términos de modelos, pensamiento interrelacionado, pensamiento dinámico y pensamiento dirigido, definidas más adelante.

Con el ánimo de presentar un estado del arte de los trabajos en el campo problemático en que se ubica esta investigación, se presentan tres niveles de actividad: las investigaciones de tipo disciplinar, las investigaciones en el ámbito de la formación básica profesional y las investigaciones e innovaciones en la educación básica. Estos tres niveles se analizan nacional e internacionalmente. Se presentan unos pocos trabajos que ilustran el estado de la investigación en el campo de los SD y del pensamiento sistémico.

En las investigaciones de tipo disciplinar se tienen los trabajos del Instituto Mediterráneo de Estudios Avanzados, a escala internacional. En el ámbito nacional están los trabajos del Grupo de Caos y Sistemas Complejos de Uniandes, Caos y Complejidad de la Universidad Nacional de Colombia, Dinusco de la Universidad Sur Colombiana, Grupo Fractales de la Universidad Industrial de Santander, entre otros.

En el campo de la formación básica profesional, está la versión online de The development of systems thinking skills using dynamics modeling tools, del profesor Ossimitz. A escala nacional está el programa del curso de Sistemas Dinámicos de la Pontificia Universidad Javeriana y el programa de Especialización en Sistemas Dinámicos de la Universidad Sergio Arboleda, entre otros.

En las investigaciones e innovaciones en educación básica están, en el plano internacional: The development of systems thinking skills using dynamics modeling tools, Systems Dynamics for Kids de Jim Hight, y The applications of Systems Dynamics Modeling in Elementary and Secondary School. A escala nacional está el Grupo de Matemáticas y Ciencias de la Escuela Pedagógica Experimental,EPE. 


\section{Contexto teórico}

Si los sistemas del mundo "real" no se parecen al mundo físico que ha construido el hombre, ha sido porque el físico se ha visto en la necesidad de fragmentar la realidad (externa) y reducir el suceso a leyes universales. Sin embargo, los fenómenos físicos del mundo que vivimos son irregulares e inestables debido a la presencia de caos, y por el contrario el mundo físico que se ha mostrado desde la física clásica es diferente del mundo vivenciado. Los sistemas físicos pueden mostrar algunas inestabilidades (sensibilidad a condiciones iniciales), haciendo que el estado futuro de estos sistemas sea impredecible en ciertas condiciones, volviéndolos complejos y derivando en sistemas caóticos (Prigogine, 1997).

La anterior reflexión nos permite volver a una intención muy clara, planteada por muchos de los docentes de ciencias, que se expresa en la necesidad de articular más significativamente el conocimiento escolar con el mundo natural en el que viven los estudiantes. Desde estos planteamientos relacionados con una "nueva racionalidad", se busca que los escolares puedan analizar los hechos donde haya inestabilidad. Para ello sólo hace falta, por un lado, desarrollar una concepción del conocimiento y del mundo natural, basado en la idea de caos-complejidad y, por otro, usar una matemática cualitativa, desde la cual se explican las irregularidades que se encuentran en los eventos físicos (Blanchard y otros, 1999).

En términos generales, el área de los sistemas dinámicos, la entendemos como la rama de las matemáticas que habla sobre el comportamiento cualitativo de sistemas que varían en el tiempo. No se trata de encontrar soluciones exactas a las ecuaciones que modelan dicho sistema (lo que frecuentemente es imposible en sistemas no lineales) sino de realizar una descripción de aspectos dinámicos del sistema. Por ejemplo, (1) si el sistema fuera estable a largo plazo, (2) si ante cambios en las condiciones iniciales hubiera variación en el estado a largo plazo del sistema, (3) si es posible alcanzar determinado estado en un tiempo fijo, o (4) si conocidas las condiciones iniciales y la naturaleza del sistema, se pudiera describir completamente la evolución del sistema (Campos e Isaza, 2002).

En este sentido, el pensamiento sistémico en torno a los sistemas dinámicos se convierte en una tendencia importante en el mundo; sin embargo, en nuestro medio han sido pocas las investigaciones desde esta forma de pensamiento en el aula. En particular, referente al pensamiento sistémico, es posible comprenderlo a partir de cuatro características, tal como lo propone Ossimitz (Ossimitz, 1997).

a. Pensamiento en términos de modelos. Es importante concientizarse de que cuando pensamos lo hacemos poniendo en juego nuestras visiones del mundo, esto es, en términos de modelos. En tal sentido, cuando pensamos sistémicamente se ponen en juego tanto la elaboración consciente de los modelos, como su validación y desarrollo posterior. En éstas son esenciales las concepciones acerca de la causalidad y las formas de representación apropiadas, como la utilización de bucles. La existencia de los bucles de recursividad es clave también en el proceso de elaboración 
de modelos ya que cuando éstos se construyen, por ejemplo en dinámicas de explicación, se elaboran con base en lo que ya sabemos, es decir, nuestra experiencia.

b. Pensamiento interrelacionado. Es una novedad frente a las formas usuales de pensar en términos de relaciones causa-efecto, que son las que han caracterizado tanto las descripciones como las explicaciones y las búsquedas en la ciencia moderna. Cuando se habla de pensamiento interrelacionado, nos encontramos con la gran riqueza que surge no sólo de la identificación de las interacciones entre las partes de un todo (sistema, por ejemplo), sino de bucles de retroalimentación que son necesarios cuando el modelo considera el tiempo. En este caso se toman en cuenta el elaborar explicaciones y modelos, efectos indirectos y redes más o menos complejas de causa y efecto, que se dan en el tiempo en una perspectiva dinámica.

c. Pensamiento dinámico. Cuando se habla de este tipo de pensamiento, se enfatiza en la posibilidad de anticiparse; se sacrifican las descripciones reversibles en las que éstas son idénticas, sea que se avance o se retroceda el tiempo, y nos adentramos en el estudio de fenómenos donde el aparecimiento del caos determinista es fuente de explicaciones en un ámbito en el que vivimos un aumento inevitable de entropía (sistemas no reversibles). En este caso, nos encontramos con emergencias inesperadas y niveles de organización. El pensamiento dinámico nos orienta hacia la elaboración de simulaciones mediante recurrencias y, con ello, a la posibilidad de contar con predicciones a corto plazo del fenómeno que se estudia.

d. Pensamiento dirigido. No se queda en la descripción sistémica sino que se preocupa por la acción orientada sistémicamente. En este sentido, cuando estamos interesados en una meta nos preguntamos por cuáles de los componentes del sistema se pueden cambiar para propiciarla. Se trata, pues, de considerar múltiples variables, incluso aquellas que hacen que nuestras ecuaciones sean tan complejas que no se les puede dar un tratamiento cuantitativo desde las ecuaciones diferenciales, sino aproximaciones cualitativas y simulaciones matemáticas.

\section{Actividades y análisis}

El estudio de la caída de los cuerpos se constituye en uno de los fenómenos físicos de gran importancia en el proyecto de Sistemas Dinámicos por las siguientes razones:

- Es un sistema físico cercano para la mayoría de las personas, en particular para los estudiantes que han abordado un curso de física (en la educación media o cursos introductorios de mecánica en la universidad), o para aquellas personas que optan por la Licenciatura en Física como profesión.

- La descripción de las ecuaciones de movimiento y la identificación del modelo matemático sobre el sistema físico permiten poner en conocimien- 
to de los estudiantes y maestros los enfoques analítico, cualitativo y numérico. El primero es el utilizado tradicionalmente en las clases de física, el segundo se describe en forma cualitativa en el plano geométrico y el tercero lo podemos utilizar para describir el evento usando el computador o la calculadora graficadora.

- El trabajo nos permite allegar elementos para mostrar la manera como los estudiantes se enfrentan a situaciones del mundo físico desde la perspectiva de los Sistemas Dinámicos, y para dimensionar nuevos aspectos con el fin de configurar la propuesta pedagógica.

Para ilustrar la vivencia del equipo con los estudiantes de la educación básica y universitaria (pregrado y posgrado), exponemos a continuación una de las situaciones analizadas en cada nivel, las ideas que se presentaron frente al evento de caída y la manera de modelar el evento. El trabajo nos permite identificar las características del pensamiento sistémico y su constitución, así como las dificultades y limitaciones en dicho pensamiento.

\section{Nivel: Educación Básica}

Se realizó el trabajo con tres instituciones públicas y una privada, IED Francisco Mirada, IED Manuelita Sáenz y la Escuela Pedagógica Experimental, EPE, con estudiantes de Educación Básica $\left(7^{\circ}, 8^{\circ} \mathrm{y}\right.$ $9^{\circ}$ ). La experiencia parte del problema práctico: ¿cómo hacer un paracaídas? Se diseñaron varios modelos de paracaídas y se experimentó enunciando las condiciones iniciales de la situación, teniendo en cuenta el sistema, las variables que influyen al caer el cuerpo (radio del paracaídas, radio del hueco interno del paracaídas, altura desde donde se deja caer, tiempo de caída, masa del objeto, forma, entre otras).

Algunas de las hipótesis o conjeturas de los estudiantes del IED Manuelita Sáenz fueron: "El tiempo de caída depende del peso del cuerpo (entre más pesado menos tiempo)". "Si cae de mayor altura, gastará más tiempo". "Entre más 'ancho' el cuerpo menos tiempo gasta" y, "todos los cuerpos caen”.

En la experiencia del evento los estudiantes opinaban lo siguiente: "El problema está generado por el aire". "No es un problema -el tiempo de caída- del peso sino la forma". "Si se pudiera quitar el aire la diferencia de tiempos de caída sería imperceptible o inexistente". "El aire empuja a los cuerpos hacia arriba" y, "a veces es muy difícil prever que pasará por la cantidad de variables". Las conclusiones que sacaron los estudiantes sobre su evento fueron: "Un sistema es un cúmulo muy complejo de factores". "Toda afirmación debe ser verificada" y, "la caída de los cuerpos, es un fenómeno físico que explica muchos eventos de la vida diaria como el movimiento del paracaídas".

El maestro titular, comenta: "Inicialmente los estudiantes ven el "sistema" solo como el cuerpo, es decir aislado del entorno. Luego hablan de interacciones "con", para referirse al "sistema" y lo "externo" al mismo, es decir lo ven como un todo. Utilizan el término variables para todo, no se logró clarificar la diferencia entre variable y parámetros, constante, etc."... "La experiencia es ampliamente constructiva, parte de las ideas y de lo que saben los estudiantes, de sus vivencias con sistemas afines, las activi- 
dades realizadas generan desequilibrios, particularmente en lo que se tenía por "cierto". La discusión entre pares y la posibilidad de experimentar, fomenta la construcción social de pensamiento, en particular aporta a la constitución del pensamiento sistémico".

En otra institución educativa, el grupo de estudiantes desarrolló la actividad enfatizando en el estudio y variación de una de las características más sobresalientes del paracaídas, descubriendo diferencias entre parámetros y variables, como lo comenta la profesora titular de la EPE:

La sesión se centró en la discusión y reflexión sobre la caída de uno de los paracaídas, al cual se puso a variar de diámetro interno. $\mathrm{Al}$ respecto se dispuso la caída del paracaídas en varios momentos, de acuerdo al nuevo diámetro del hueco. La observación se centró tanto en el tiempo de caída, como en el trayecto del paracaídas. Los estudiantes lanzaron hipótesis de acuerdo a la situación y se observó también la capacidad que tienen los muchachos para establecer relaciones entre las variables y parámetros de la situación.

Se utilizó el paracaídas de una estudiante, lanzado siempre desde una altura de 2,76 m. Inicialmente, con un diámetro interno de $12 \mathrm{~cm}$, los tiempos de caída fueron 1'27 segundos y 1'50 segundos; posteriormente se amplió este diámetro a $14 \mathrm{~cm}$ y el tiempo de caída fue 1'13 segundos. De acuerdo con estos y otros datos, se infiere:

- El paracaídas de menor diámetro interno cae más lento.

- El paracaídas con mayor diámetro se abre al principio, y al final tiene una caída extraña (aleatoria, caótica).
- Con el paracaídas de mayor diámetro del hueco se cumple también que hasta la mitad cae más lento y después de la mitad cae más rápido.

Variables y parámetros relacionados con la caída del paracaídas: diámetro interno, altura de lanzamiento, tiempo de caída, diámetro externo y masa que cae.

A partir del cuestionamiento sobre la incidencia del diámetro interno (hueco), se lanzaron las siguientes hipótesis:

- Si no hay hueco (diámetro interno $=0$ ), el paracaídas actúa como una cometa.

- El diámetro del hueco que permite que aún siga siendo paracaídas iría hasta la mitad del radio externo (cuando se pase de esta medida, ya no cumpliría condiciones de paracaídas).

- El paracaídas necesita atrapar aire. Las relaciones que los estudiantes lograr establecer para el caso del paracaídas son éstas: A mayor diámetro externo, mayor tiempo, es decir, que a mayor superficie circular del paracaídas mayor es el tiempo que tarda en llegar al suelo. Por otra parte, a mayor diámetro del hueco, menor será la superficie de contacto con el aire y por tanto será menor el tiempo de caída. Los estudiantes hacen el estudio experimental y observan que esto ocurre así, hasta que el hueco llega a tener un diámetro igual a la mitad del diámetro del paracaídas.

Diámetro interno $D$, diámetro externo $d$. $d \alpha t$

$D \propto 1 / t\left(d_{i} \geq \mathrm{D} / 2\right)$

A mayor altura de lanzamiento, mayor tiempo de caída $h \alpha t$

A mayor masa (que cae), menor tiempo de caída $\quad m \alpha 1 / t$ 
Como se observa en un proceso de construcción del fenómeno de caída, como el del paracaídas, los estudiantes pueden relacionar variables algebraicas de proporcionalidad, modelar matemáticamente dicho fenómeno y luego pueden llevar estas relaciones de variables y parámetros al computador para iniciar un proceso de simulación.

\section{Nivel: Licenciatura en Física del Departamento de Física de la Universidad Pedagógica Nacional}

El trabajo se desarrolló en el Seminario de Línea del equipo de sistemas dinámicos, en el que participaban los estudiantes inscritos en la línea (10) y los maestros coordinadores de la investigación (3). Se partió del conocimiento que los estudiantes tenían sobre la caída de los cuerpos y se invitó a cada uno a presentar la situación y el modelo matemático desde el cual se podían dar cuenta de su comportamiento. Para ello se les solicitó definir el sistema de estudio, las variables y los parámetros que describían su sistema, aspectos que permiten explicitar el sistema, sus interacciones y relaciones.

Los alumnos representaron el fenómeno en el computador y estudiaron la información de las gráficas y las tablas de datos que obtuvieron analizando su comportamiento o relaciones. Algunas de las situaciones presentadas por los estudiantes fueron:

Quise considerar una variable tan común como la presencia del aire y me empecé a dar cuenta del efecto que produce ésta en la caída de un objeto cualquiera. Pensé que en un modelo completamente ideal el aire aparece inexistente pero que sí éste se consideraba, entonces debía pro- ducir un efecto de resistencia porque a medida que el cuerpo va cayendo, el aire va a retardar el movimiento; en este sentido el aire comportaría, en términos de fuerzas, como una fuerza de fricción para el movimiento. Bien, obvié el tamaño, forma del cuerpo cayendo, temperatura y densidad del aire, ya que serían otras variables más que se tendrían en cuenta y esto complejisaría' la situación.

Otra, tenía que ver con la intención explícita de considerar el aire como elemento de interacción:

Para considerar la caída de un cuerpo verticalmente en un medio con rozamiento, para este caso tendremos que hacer unas consideraciones adicionales. La primera y básica, considerar la fuerza de rozamiento $f$, proporcional a la velocidad. Estas variables las podemos relacionar con dos variables más, una de ellas llamada $K$, que es el coeficiente de fricción $y$ que depende de la forma del cuerpo, la otra variable $\beta$, coeficiente de viscosidad y que depende del medio. En este caso como el medio es el aire, $\beta$ va a depender ahora de la temperatura del medio, llegando a la relación $\beta=f(\mathrm{~T})$. T, temperatura.

Esta manera de abordar la situación de estudio nos pone de presente la necesidad de explicitar el fenómeno estudiado, sus condiciones y relaciones, la forma como se describe la dinámica del sistema, los parámetros que hay que tener en cuenta y los posibles acontecimientos. Modo de proceder que nos instaura en otra manera de ver los sistemas físicos, pues lo importante no

1 Es el término que utiliza el estudiante. Más apropiado sería "y esto volvería más compleja la situación". 
es plantear desde el principio una(s) ecuación(es) de movimiento, remplazar datos y hallar una respuesta, sino más bien comprender lo que cada uno realiza y cómo se piensa el fenómeno físico, describiendo desde el comienzo el sistema con el mayor detalle posible, lo cual nos permite visualizar el tipo de interacciones sobre éste, las relaciones entre las variables y qué predicciones se pueden hacer, para plantear posteriormente la(s) ecuación(es) de movimiento y hacer la modelación física del evento.

A continuación mostramos el proceder, la forma de relacionar las variables y parámetros del evento, las consideraciones y la descripción que realizan tres de los estudiantes de la licenciatura.

Plantean que los objetos cercanos a la superficie de la Tierra tienen una aceleración constante $g$. Si la aceleración es la derivada de la velocidad, se tiene que: $d v / d t=a \mathrm{Y}$; tomando como positiva la dirección hacia abajo y la aceleración igual a $g$, se obtiene: $d v / d t=g$. Esta última ecuación diferencial nos da cuenta de la variación de la velocidad del cuerpo al caer. Si, además, suponemos que el cuerpo es lanzado con una velocidad inicial $v_{0}$, debe resolverse: $d v / d t=g$; $0<t<t_{1}: s^{\prime}$ $(0)=v_{0}$, tomando $t=0$ como el instante inicial y $t_{1}$ el tiempo que transcurre hasta que el cuerpo cae a la Tierra.

Sabemos, por la mecánica newtoniana, que la fuerza ejercida sobre un cuerpo es proporcional a la aceleración producida en éste, por lo que se tiene: $F \alpha a \rightarrow F=m a$; podemos escribir esta expresión en la siguiente forma: $m a=$ $m d v / d t$.

En la caída libre, cuando no se considera un caso ideal en las condiciones del problema, los cuerpos se ven afectados por distintos factores que pueden influir en su movimiento: por ejemplo, la fricción que ejerce el medio a través del cual cae (aire), las corrientes que ocurren en éste, la presión a la que está sometido, entre otros.

Si se quiere ver cómo influye sólo la fuerza de fricción que ejerce el aire, se debe considerar a éste como un fluido, en el cual dicha fuerza puede ser proporcional a la velocidad del objeto y opuesta a ella, entonces se puede escribir la fuerza debida a la fricción como: $F_{f}=-k v$. Ahora bien, como sobre el cuerpo está actuando también el campo gravitatorio (peso del cuerpo $w$ ), tenemos: $w=m g$.

Para el cuerpo, la fuerza total aplicada será entonces $F_{\mathrm{T}}=w+F_{f}$. Tomando como positiva la dirección hacia abajo, es posible escribir la expresión:

$m a=m g-k v$

$\mathrm{y}$, utilizando: $m a=m d v / d t$, tenemos que:

$m d v / d t=m g-k v$ ó $d v / d t+(k / m) v=g$

Así, para modelar la velocidad de la caída de un cuerpo, se tienen en cuenta las siguientes hipótesis: "Sobre el cuerpo actúa una fuerza $w$ constante"; "la aceleración produce un aumento constante de la velocidad" y "existe una fuerza de fricción que resiste el movimiento generada por el aire, que crece al aumentar la velocidad del cuerpo". Las variables implicadas son:

$t \rightarrow$ tiempo (variable independiente); $v \rightarrow$ velocidad (variable dependiente); 
$m \rightarrow$ masa (parámetro); $g \rightarrow$ gravedad (parámetro) y, $k \rightarrow$ coeficiente de fricción (parámetro).

Adecuación de las variables dependientes, independientes y los parámetros definidos en la ecuación diferencial del fenómeno de caída del cuerpo: Variable independiente $t$; variable dependiente $v y$, parámetros $k, m, g=9,8 \mathrm{~m} / \mathrm{s}^{2}$.

Analíticamente la solución de este sistema está dada por:

$v(t)=v_{0} \mathrm{e}^{-k t / m}+m g / k\left(1-\mathrm{e}^{-k t / m}\right)$

En este trabajo se avanza en considerar la resistencia del aire, introduciendo más parámetros en la ecuación. Esta consideración se ajusta más al suceso real, pero para contemplar el suceso con más aproximación es necesario tener en cuenta también que el aire depende de la temperatura y presión, además de la convección.

\section{Nivel: Maestría en Docencia de la Física, Departamento de Física de la Universidad Pedagógica Nacional}

En el contexto del seminario de Actualización, Módulo de Tópicos de Física de la Maestría en Física de la UPN, se aborda el estudio de los sistemas dinámicos desde los presupuestos epistemológicos, didáctico-pedagógicos y disciplinares. Particularmente se aborda el estudio de la caída de los cuerpos con los maestros del curso y se realiza el estudio utilizando el computador, para lo cual se acude al programa Excel. Se hace la simulación del evento en dos situaciones a saber: 1) La caída de un cuerpo teniendo en cuenta únicamente su interacción gravitacional y las condiciones iniciales del evento, y 2) La caída de un cuerpo teniendo en cuenta el medio con el cual interactúa, el cuerpo que cae, sus dimensiones, el área de contacto, entre otros.

A continuación se exponen apartes del trabajo en cada situación, realizadas por Fabio Arcos, uno de los profesores participante en la maestría, quién dice:

La modelación de un problema físico, que me ha causado bastante curiosidad, es el de la caída de los cuerpos. En mi estudio particular busco establecer cómo obtener el valor de la velocidad límite para cualquier objeto que cae, en cualquier medio en el que se presente la caída. Una de las razones en las que estriba esta preocupación consiste en poder ilustrar que los movimientos con velocidad constante, que usualmente se consideran ideales, hacen parte de los problemas reales, que nos permiten comprender por qué el granizo no nos rompe la cabeza cuando cae.

Primera situación: Caída libre sin fricción a la que se le puede variar la velocidad inicial; aquí no hay velocidad límite para los objetos que caen.

\begin{tabular}{|c|c|c|c|c|c|}
\hline & & & Gravedad & $-9,8$ & $\mathrm{~m} / \mathrm{s}^{\wedge}{ }^{2}$ \\
\hline & & & Velocidad inicial & 50 & $\mathrm{~m} / \mathrm{s}$ \\
\hline Tiempo (s) & Posición (m) & Velocidad $(\mathrm{m} / \mathrm{s})$ & Delta t & 0,05 & $\mathrm{~s}$ \\
\hline 0 & 100 & 50 & Altura & 100 & $\mathrm{~m}$ \\
\hline
\end{tabular}



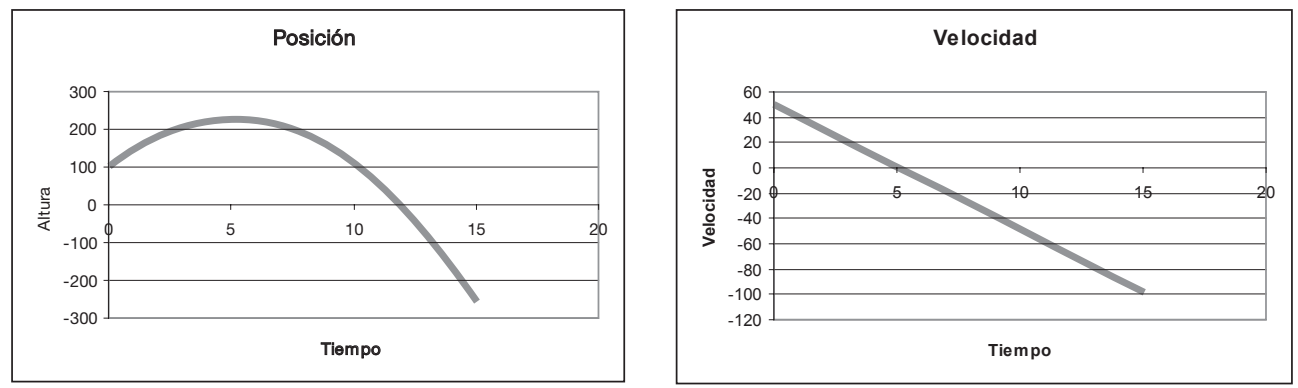

En la gráfica de la izquierda se observa la caída de un objeto con velocidad inicial hacia arriba y a la derecha se muestra cómo la velocidad disminuye uniformemente. Gráficas que encontramos por lo general en los textos escolares.

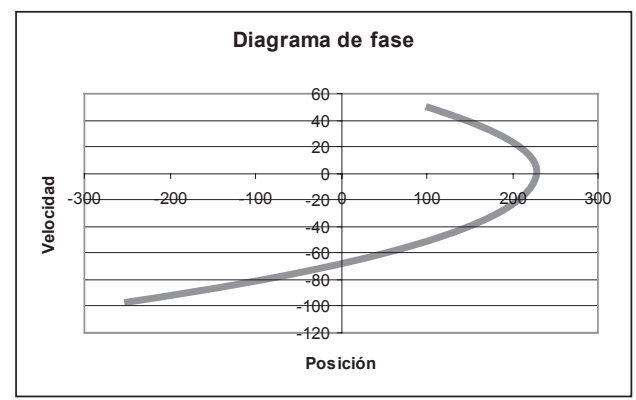

En este espacio de fase se muestra cómo la velocidad disminuye y se representa por una parábola acostada.

Segunda situación: Velocidad límite para cualquier objeto que se deja caer en cualquier medio.

El profesor quiere tener en cuenta que la velocidad constante sí se puede lograr, aun experimentalmente, y no es un caso ideal. Para ello considera el peso, la fuerza de rozamiento proporcional al cuadrado de la velocidad y el empuje por estar en un fluido.

Toma en cuenta la forma, tamaño y peso del objeto que cae para hacer un cálculo de $\varepsilon$ y de la velocidad límite. Si bien se trata de ecuaciones diferenciales que se pueden resolver analíticamente, realiza una hoja de cálculo dinámica en la que se pueden visualizar las variables como funciones del tiempo y el diagrama de fase. El usuario de esta hoja puede modificar los valores de entrada (cuarta y quinta columnas de la siguiente tabla) y las condiciones iniciales (para $t=0$ ), obteniendo diferentes comportamientos.

\begin{tabular}{|c|c|c|c|c|c|}
\hline & & & Gravedad $g$ & 9,8 & $\mathrm{~m} / \mathrm{s}^{2}$ \\
\hline & & & Densidad $\rho m$ & 1,3 & $\mathrm{~kg} / \mathrm{m} 3$ \\
\hline Tiempo $(\mathrm{s})$ & Posición $(\mathrm{m})$ & Velocidad $(\mathrm{m} / \mathrm{s})$ & Densidad objeto $\rho$ & 850 & $\mathrm{~kg} / \mathrm{m} 3$ \\
\hline 0 & 100 & 30 & Coeficiente de arrastre $D$ & 0,6 & \\
\hline 0,1 & 103 & 29,43444 & Área del objeto $A$ & 0,01 & $\mathrm{~m}^{2}$ \\
\hline 0,2 & 105,943444 & 28,85345722 & Masa del objeto $m$ & 0,85 & $\mathrm{~kg}$ \\
\hline 0,3 & 108,8287897 & 28,25693673 & Longitud del objeto $/$ & 0,1 & $\mathrm{~m}$ \\
\hline 0,4 & 111,6544834 & 27,64478525 & Delta $t$ & 0,1 & $\mathrm{~s}$ \\
\hline
\end{tabular}


Las siguientes ecuaciones relacionan

los diferentes parámetros del sistema

$$
G=\left(\frac{\rho_{m}}{\rho}-1\right) g \quad \varepsilon=\frac{\rho_{m} D A}{2 m} \quad v_{L}=\sqrt{\frac{-G}{\varepsilon}}
$$

\begin{tabular}{|c|c|c|c|c|c|c|c|c|c|}
\hline $\mathbf{G}$ & $-9,785$ & $\mathrm{~m} / \mathrm{s}^{2}$ & $\boldsymbol{\varepsilon}$ & 0,004588 & $\mathrm{~m}-1$ & $\mathrm{~V}_{\mathrm{L}}$ & 46,180415 & $\mathrm{~m} / \mathrm{s}$ & $0,2<\mathrm{D} \leq 2$ \\
\hline
\end{tabular}
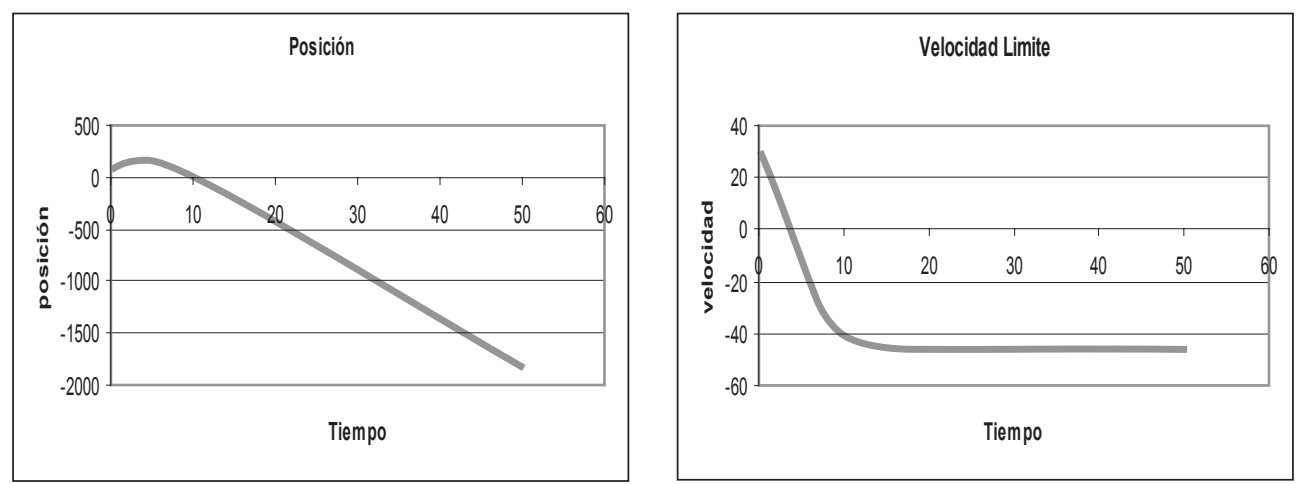

En la gráfica de la izquierda se observa cómo la posición varía primero casi como una parábola y después uniformemente (a partir de los $10 \mathrm{~s}$, aprox.), porque alcanza una velocidad límite, como se muestra en la gráfica de la derecha.

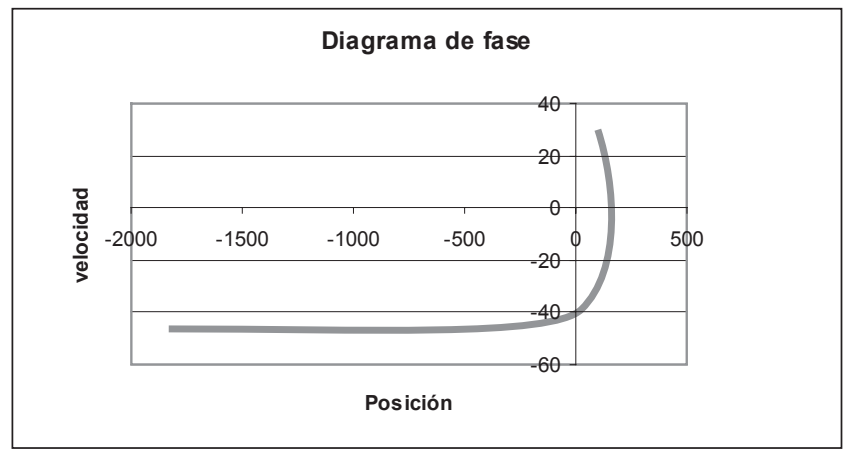

En este diagrama de fase se observa cómo, dependiendo del medio en el que el cuerpo cae, la velocidad límite se alcanza en mayor o menor tiempo.

En lo referente al análisis de esta actividad, vale la pena mencionar que aproximarse a la modelación de los problemas reales que gobiernan una canti- dad en una ecuación diferencial y poder predecir el valor futuro de esta cantidad es un ejercicio que requiere tiempo y es de gran importancia en la descripción 
de los eventos físicos. Además, retomar el significado de las ecuaciones diferenciales, sus problemas, sus formas de solución, así como las aproximaciones a soluciones que ofrecen los métodos numéricos, son aspectos significativos para modelar el fenómeno físico, y recurrir al computador para simular el evento (programa Excel) se constituye en una herramienta que permite ver la evolución y representación del fenómeno en estudio.

\section{Reflexiones finales}

\section{En cuanto a la mirada general sobre la enseñanza de la física}

En primer lugar, encontramos que en las clases de física de enseñanza media y en los cursos introductorios de mecánica en el ámbito universitario, se aborda el estudio del evento de la caída de los cuerpos. La descripción y la explicación de lo que acontece se reducen a la presentación de fórmulas sobre el tiro vertical hacia abajo o hacia arriba:

$$
\begin{aligned}
& v f=v i+g t \\
& h=v i t+1 / 2 g t^{2} \\
& v f^{2}=v i^{2}+2 g h
\end{aligned}
$$$$
v f=g t
$$

$v f=v i-g t$

$h=v i t-1 / 2 g t^{2}$

$v f^{2}=2 g h$
Donde $v_{f}$ es la velocidad final, $v_{i}$ la velocidad inicial, $g$ la gravedad $(9,8 \mathrm{~m} /$ $\left.\mathrm{s}^{2}\right), t$ el tiempo y $h$ altura.

El estudio de la caída de los cuerpos se limita a la realización de ejercicios varios, donde a partir de unos datos, $v_{i}$ igual a cero (cuando se deja "caer libremente" el cuerpo o parte del reposo), se varía la altura de la cual se deja caer el cuerpo, por ejemplo. El estudiante lo único que tiene que hacer es "aprenderse” las fórmulas, manejar adecuadamente el algoritmo reemplazando los datos en la ecuación y hallar una respuesta numérica. En últimas, el evento se reduce a remplazar valores y realizar unos procedimientos algebraicos dados en la ecuación, el estudio físico nunca se presenta, como la descripción de la dinámica del evento, las explicaciones y su modelar físico.

Esta situación se presenta porque los maestros sólo conocen esta manera de abordar las situaciones físicas o porque fue la forma como les enseñaron y "repiten" este estilo, que a la vez se expone en los textos escolares de física. Otra posibilidad es que al considerar nuevos parámetros en las ecuaciones de movimiento, la solución de la ecuación no es tan fácil de abordar desde los métodos analíticos y se tiene que recurrir a otros métodos, como la técnica de los métodos numéricos (llevados al computador) o de los cualitativos (descripciones geométricas). Estos dos últimos métodos cobran sentido en la matemática contemporánea, particularmente de los sistemas dinámicos.

\section{En cuanto al evento de caída de los cuerpos}

El estudio de la caída de los cuerpos, como se presentó en las experiencias con los distintos niveles de escolaridad que aquí se abordaron, nos demuestra cómo 
la caída de un cuerpo está conectada de múltiples maneras con su entorno y, en última instancia, con el resto del universo. Sin embargo, no importa cuántas conexiones tomemos en cuenta para describir un fenómeno, siempre estaremos obligados a excluir otras (como nos lo dice Capra, 1998).

Por otra parte, la vivencia nos posibilita ubicar que las diversas maneras de pensar el fenómeno por parte de los estudiantes, las múltiples formas de abordarlo y las consideraciones que realizan de acuerdo con la situación que eligen, enriquecen su pensamiento y les permite vivir el estudio de la caída de los cuerpos y darse cuenta de que es posible aprender de formas alternativas, aprender del otro cuando intercambian ideas y profundizar en las ecuaciones de movimiento, así se comprende la actividad de modelar. Situación que a la vez que nos instaura en otra manera de asumir el estudio de los sistemas físicos, nos posibilita dimensionar la perspectiva de los sistemas dinámicos desde enfoques no solamente analíticos sino también numéricos y cualitativos propios del conocimiento contemporáneo (Blanchard et al., 1999).

\section{Relacionadas con la perspectiva de los sistemas dinámicos y la modelación de los eventos físicos}

Los sistemas dinámicos como herramienta matemática nos proveen de un proceder que nos permite acercarnos a realizar descripciones más detalladas de los fenómenos físicos, que de alguna manera abren paso a una infinidad de acercamientos e interacciones que la descripción tradicional no toma en cuenta por la imagen que se tiene de natura- leza, como un sistema lineal, simétrico y reversible, entre otros aspectos.

Aproximarse a la modelación de problemas reales, es decir, convertir las reglas que gobiernan una cantidad en una ecuación diferencial y poder predecir el valor futuro de esta cantidad, es un ejercicio que requiere tiempo $y$ dedicación. Retomar el significado de las ecuaciones diferenciales, sus problemas, sus formas de solución, así como las aproximaciones a soluciones que ofrecen los métodos numéricos, son, entre otros, los aspectos más significativos en la actividad de la modelación de eventos físicos como el de la caída de los cuerpos y el péndulo, que con ayuda de los computadores se constituye en una herramienta potente en dichas aproximaciones. Por ejemplo, la caída que experimenta un cuerpo puede verse de varias maneras. Una de éstas sería despojarla de cualquier nexo de ocurrencia y considerar sólo su interacción gravitacional y las condiciones iniciales de dicho movimiento. Esto es lo que se denomina una caída libre y es el único sistema que se aborda en la enseñanza tradicional básica y media.

Otras posibilidades de abordar la situación se presentan cuando se toman en cuenta el medio con el que se interactúa, el cuerpo que cae, sus dimensiones, el área de contacto, entre otros. El considerar o no cada uno de estos parámetros conduce a modelos diferentes.

\section{Relacionadas con la caracterización del pensamiento sistémico}

El cambio del pensamiento reduccionista al pensamiento sistémico se genera cuando se trata de incrementar la descripción de un fenómeno como 
el de la caída de los cuerpos. El nuevo pensamiento sistémico, la metáfora del conocimiento como construcción, se remplaza por la de red. Al percibir la realidad como una red de relaciones, nuestras descripciones forman también una red interconectada de conceptos y modelos en la que existen cimientos. Por ejemplo, en el estudio de la caída efectuada en el proceso investigativo se muestran múltiples tipos de interacciones del sistema con su entorno y en última instancia, con el resto del universo. Sin embargo, no importa cuántas conexiones tomemos en consideración para describir el fenómeno, siempre estaremos obligados a excluir otras (Capra, 1998).

\section{Bibliografía}

Blanchard, P., Devaney, R., Hall, G. (1999). Ecuaciones Diferenciales. México: International Thomson Editores.

Campos, D. e Isaza, J. (2002). Prolegómenos a los sistemas dinámicos. Bogotá: Universidad Nacional de Colombia, Colección de Textos.

Capra, F. (1998). La trama de la vida. Una nueva perspectiva de los sistemas
Además, para el caso de la enseñanza de la física, nos permiten mostrar que si se parte de una situación abierta, los estudiantes identificarán el sistema, los parámetros con los cuales van a plantear la(s) ecuación(es) de movimiento y su modelar físico. En este sentido, como se expone en los relatos de los estudiantes en los tres niveles de educación, se fortalecen los rasgos del pensamiento sistémico, lo cual se observa no sólo por la capacidad de modelar, es decir, de armar sus propias relaciones entre variables y parámetros, o por llevarla al computador para obtener gráficas, sino fundamentalmente por la comprensión de la iteración que está en la base del estudio de los fenómenos abordados. $\boldsymbol{\Delta}$

vivos. Barcelona: Editorial Anagrama, Colección Argumentos.

Ossimitz, G. (1997). The Development of Systems Thinking Skills Using System Dynamics Modeling Tools. http:// wwwu.uni-klu.ac.at/gossimit/sdyn/ gdm_eng.htm

Prigogine, I. (1997). El fin de las certidumbres. Madrid: Taurus-Grupo Santillana. 